Revista de Biología Marina y Oceanografía

Vol. 48, №3: 667-672, diciembre 2013

DOI 10.4067/S0718-19572013000300025

Nota Científica

\title{
Estado del conocimiento de los piqueros pantropicales (Sulidae) en las islas oceánicas chilenas y primer registro de piquero café Sula leucogaster en Isla de Pascua
}

State of knowledge of Pantropical Boobies (Sulidae) in Chilean oceanic islands and first record of the Brown Booby Sula leucogaster in Easter Island

\author{
Marcelo Flores ${ }^{1}$, Pedro Lazo² y Rodrigo Hucke-Gaete ${ }^{3}$
}

\author{
'Departamento de Ecología y Biodiversidad, Facultad de Ecología y Recursos Naturales, Universidad Andres Bello, República \\ 470, Piso 3, Santiago, Chile. mflores.24@gmail.com \\ ${ }^{2}$ Corporación Nacional Forestal, CONAF, Mataveri s/n, Isla de Pascua, Chile \\ ${ }^{3}$ Instituto de Ciencias Marinas y Limnológicas, Universidad Austral de Chile, Casilla 567, Valdivia, Chile
}

\begin{abstract}
We present a review of the current state of knowledge of the Pantropical Boobies, namely: the Red-footed Booby (Sula sula), the Brown Booby (S. leucogaster) and the Masked Booby (S. dactylatra) throughout the Chilean oceanic islands. The lack of information on this group means that it is not possible to accurately determine the current status of these species in Chilean waters. It is thus imperative to increase monitoring effort and develop research projects in order to fill the knowledge gaps for these species in particular and insular avifauna in general. Finally, the first documented record of Brown Booby in Chile is reported.
\end{abstract}

Key words: Pantropical Boobies, Sula leucogaster, Easter Island, Chile

\section{INTRODUCCIÓN}

De acuerdo con Ainley (1980), 9 de las 156 familias de aves del mundo están especializadas como aves marinas. Dentro de éstas, se encuentra la familia Sulidae, que comprende los géneros Sula (piqueros) y Morus (alcatraces), pertenecientes a un orden ancestral que probablemente se originó hace más de 60 millones de años. En el género Sula, se encuentra un grupo de piqueros denominados piqueros pantropicales, que incluyen al piquero café (Sula leucogaster), al piquero blanco ( $S$. dactylatra) y al piquero de patas rojas ( $S$. sula) (Carboneras 1992). Estas especies, de amplia distribución en zonas tropicales y correlacionadas con la distribución de presas como peces voladores (Murphy 1936), se han adaptado a vivir en ambientes similares y poseen distribuciones e historias de vida paralelas (Carboneras 1992).

El estado del conocimiento de la ornitofauna de las islas oceánicas chilenas es escaso, aun cuando las especies que habitan estas islas son relativamente conocidas a nivel mundial, se mantiene lo indicado por Schlatter (1984) hace casi 3 décadas: se conoce muy poco a nivel local sobre su abundancia relativa, biología y estado actual, especialmente en aquellas islas de más difícil acceso. Schlatter (1987) hizo un diagnóstico de esta problemática indicando que el estado ecológico de las islas es desalentador. Por lo anterior, el objetivo del presente trabajo fue determinar el estado de conocimiento de los piqueros pantropicales que habitan en las islas oceánicas de Chile.

\section{MATERIALES Y MÉTODOS}

Se realizó una búsqueda de información bibliográfica sobre aves de la familia Sulidae presentes en las islas oceánicas chilenas a través de servidores y bases de datos en internet, consultando y recopilando referencias y material bibliográfico en la biblioteca de la Facultad de Ciencias del Mar y de Recursos Naturales de la Universidad de Valparaíso, biblioteca William Mulloy del Museo Antropológico Padre Sebastian Englert en Rapa Nui, bibliotecas privadas de ornitólogos nacionales y extranjeros, archivos de la oficina provincial de la Corporación Nacional Forestal (CONAF) en Isla de Pascua y entrevistas a guardaparques e isleños en un viaje a Isla de Pascua realizado entre el 10 y 21 de noviembre de 2012, donde se realizaron recorridos por la isla y se visitaron los islotes Motu Nui e Iti para realizar conteos de aves marinas. Se tomaron fotografías de algunas aves para su reconocimiento y se revisó la información bibliográfica 


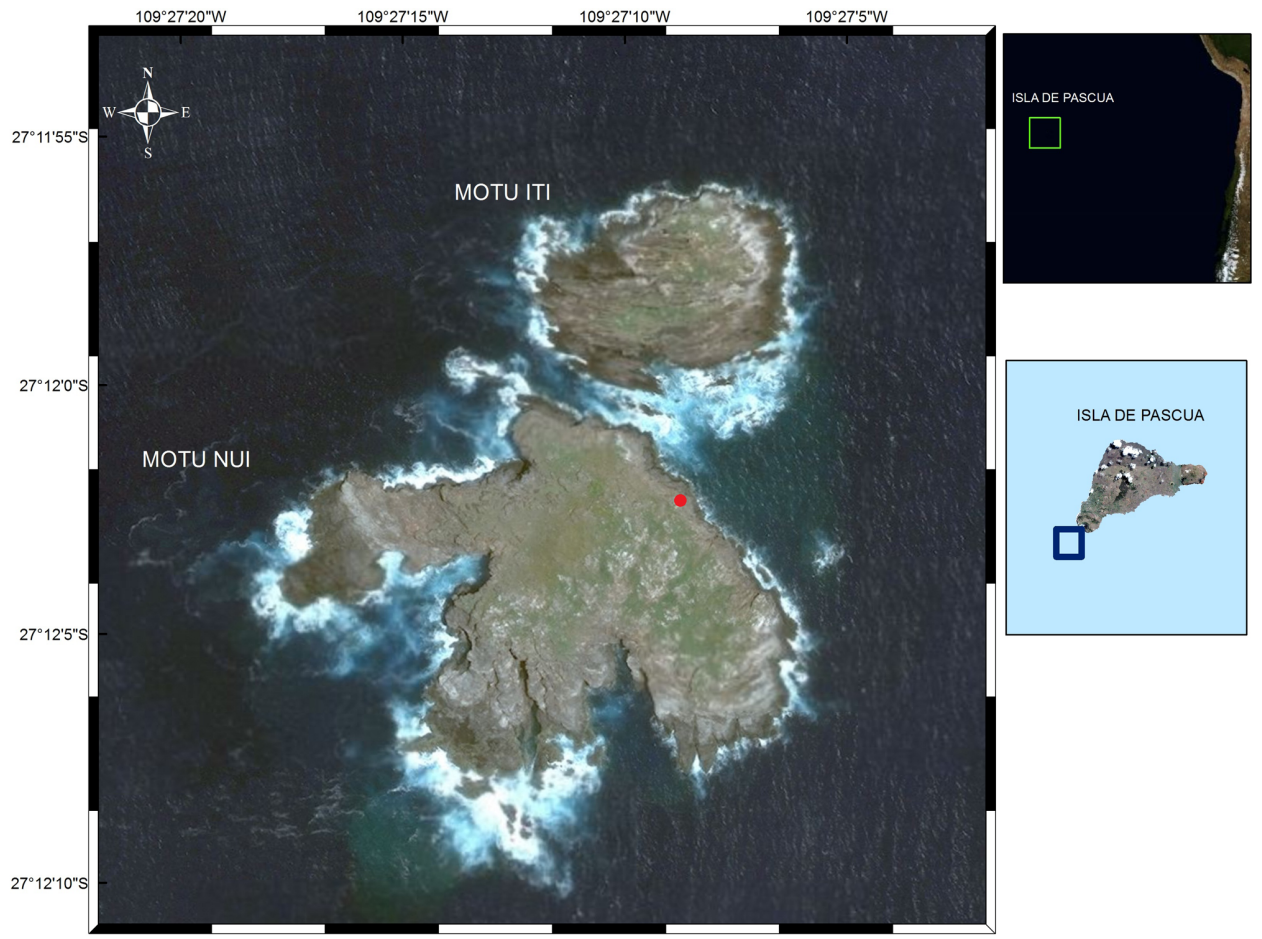

Figura 1. Posición del avistamiento de piquero café Sula leucogaster en Motu Nui, Isla de Pascua (punto rojo). Imagen: Jaime Aguilera $\odot$ / Position of the sighting of Brown Booby Sula leucogaster in Motu Nui, Easter Island (red dot). Image: Jaime Aguilera $\odot$

para sintetizar y evaluar los reportes especialmente de avistamientos y estudios científicos realizados en los piqueros pantropicales.

\section{Resultados y Discusión}

\section{Piquero de patas rojas Sula Sula (Linnaeus, 1766)}

El piquero de patas rojas ha sido reportado para la isla Salas y Gómez y recientemente para Isla de Pascua, totalizando 3 registros en el país. Los registros en isla Salas y Gómez fueron realizados por Harrison \& Jehl (1988), que mencionan la presencia de un individuo juvenil en marzo de 1985. Luego, 10 años después, Vilina \& Gazitúa (1998) informan la presencia de un adulto y un juvenil en diciembre de 1997. El registro en Isla de Pascua, corresponde a un ejemplar juvenil observado en Motu Nui, en febrero de 2010, que aparentemente permaneció por aproximadamente 2 meses en el sector (GonzálezAcuña et al. 2010). Recientemente, Lazo (P. Lazo, datos no publicados) registró la presencia de 2 ejemplares de fase clara en el Motu Iti.
La escasez de registros, permiten suponer que se trata de una especie visitante dado que posee una amplia distribución en mares tropicales y subtropicales planeta nivel global (King 1967, Harrison 1991) y presenta la mayor distribución de todos los representantes de la familia Sulidae ya que puede realizar recorridos entre islas (Nelson 2005), desplazarse durante la noche (Carboneras 1992) y utilizar corrientes de aire para planear (Weimerskirch et al. 2005). Si bien ambas islas reúnen los requisitos necesarios para la nidificación, ya que puede hacerlo sobre la vegetación, suelo o acantilados (Shallenberger et al. 1974, Carboneras 1992), la presencia de juveniles, pero no de nidos o pollos, no permiten establecer su grado de residencia. Es posible que el piquero de patas rojas registrado en las islas oceánicas, corresponda a la subespecie Sula sula websteri, de amplia distribución en el Pacífico Este (Carboneras 1992, Schreiber et al. 1996), siendo el registro más cercano en islas Gambier, a ca., 2.600 km de Salas y Gómez (Thibault \& Bretagnolle 1999). 


\section{Piquero café Sula leucogaster (Boddaert, 1733)}

El piquero café ha sido reportado para las aguas frente a las costas entre Arica y Antofagasta (Millie et al. 1973) y para la isla Salas y Gómez; este último avistamiento aparece en la 'Guía de Campo de las Aves de Chile' (Araya \& Millie 1986), sin embargo en dicha publicación no se entregan detalles.

El 14 de noviembre de 2012, se accedió al grupo de islotes (motus) ubicados al SW de la Isla de Pascua, permaneciendo por un periodo de $8 \mathrm{~h}$ en Motu Nui. Luego de registrar la presencia de las aves en el lugar, aproximadamente a las 17:30 h, se observó y fotografió un ejemplar adulto de piquero café sobrevolando el motu para luego posarse en la parte este del mismo (Fig. 1). Las fotografías fueron analizadas y se constató la identificación inicial in situ utilizando las guías de identificación de aves de Harrison (1991) y Jaramillo (2005). La Figura 2 confirma que se trata de un piquero café, que se caracteriza por ser un piquero mediano, con el adulto de color café en la parte dorsal y cuello pero con las partes inferiores blancas. Posee una división nítida entre el pecho oscuro y las partes inferiores blancas a nivel del borde anterior del ala en vuelo. La cara inferior del ala interna es blanca con una raya oscura en el cúbito. Los ojos y el pico son de color amarillo, mientras que los pies son de un color amarillo más claro. Aunque puede confundirse con el juvenil de piquero blanco, debido a la nítida división entre la cabeza oscura y las partes inferiores blancas, la transición oscuro/claro en el piquero blanco juvenil está en el cuello, mientras que en el piquero café se encuentra en la línea que va del pecho al borde anterior del ala (Harrison 1991, Jaramillo 2005).

La información existente para la especie presenta imprecisiones que no permiten determinar la presencia para la especie en Chile. El registro en isla Salas y Gómez, no ha sido considerado en ninguna publicación posterior a la guía de campo de Araya \& Millie (1986). Considerando que esta especie posee una gran similitud con el juvenil de piquero blanco (Sula dactylatra), podría tratarse de una identificación errónea, como también lo sugiere Martínez \& González (2004). El trabajo realizado por Millie et al. (1973) incluye varios avistamientos, sin embargo, no existe certeza de la ubicación de éstos ya que un primer avistamiento se reporta $c a$., $13 \mathrm{~km}$ al norte del reconocido límite marítimo con Perú, ubicado en los $18^{\circ} 21^{\prime} \mathrm{S}$. El segundo reporte indica el registro de 31 ejemplares y 6 avistamientos. Esta área incluye aguas peruanas por lo que no es posible saber cuántos avistamientos ocurrieron efectivamente en aguas chilenas, aun cuando a lo menos un ejemplar debe haber sido visto en la latitud $23^{\circ} 55^{\prime} \mathrm{S}$. Dado que existen reportes para la especie en aguas peruanas (Valverde 2007), los registros de Millie et al.

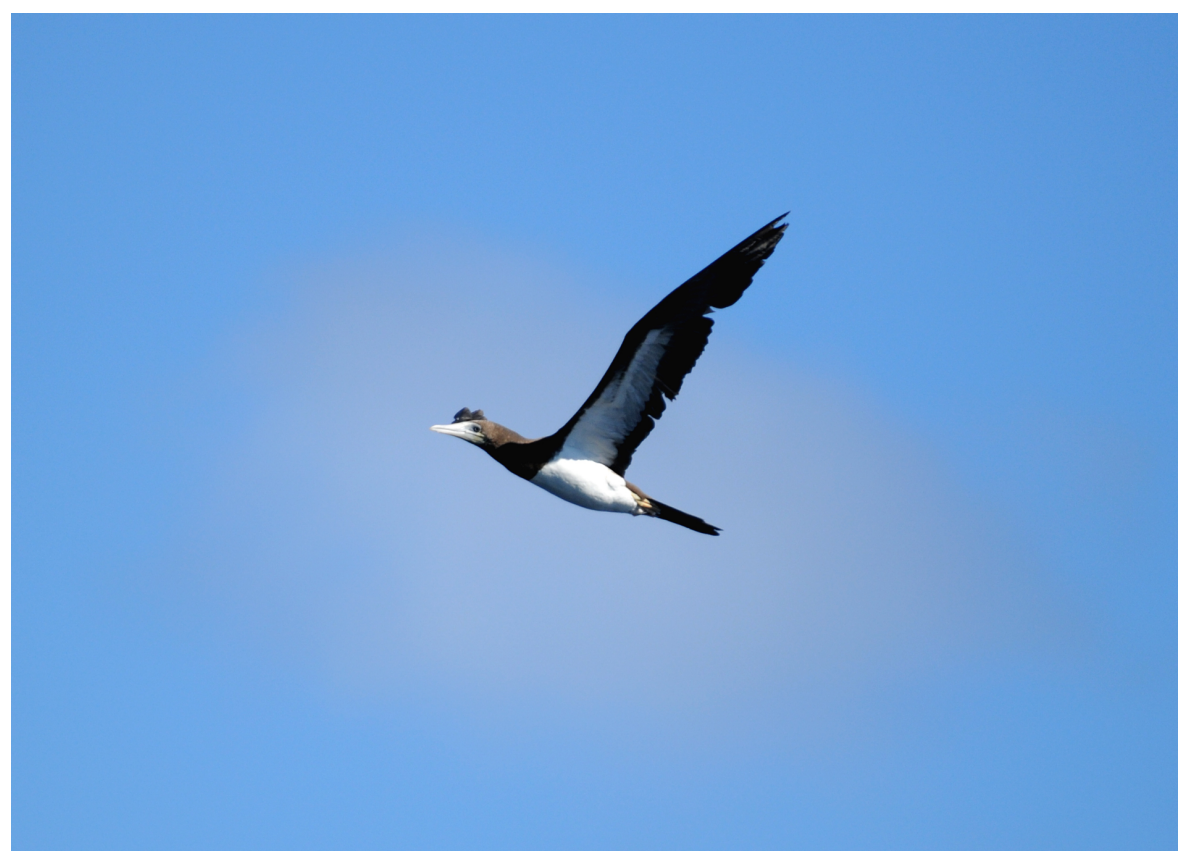

Figura 2. Ejemplar adulto de piquero café Sula leucogaster, sobrevolando el Motu Nui, Isla de Pascua, durante noviembre 2012. Fotografía: Rodrigo Hucke-Gaete $\odot$ / Adult of Brown Booby Sula leucogaster, flying over Motu Nui, Easter Island during November 2012. Photo: Rodrigo Hucke-Gaete ( 
Tabla 1. Datos cuantitativos de piquero blanco Sula dactylatra en las islas oceánicas chilenas: Desventuradas (San Félix y San Ambrosio), isla Salas y Gómez e Isla de Pascua / Quantitative data of Masked Booby Sula dactylatra in the Chilean oceanic islands: Desventuradas Islands (San Félix and San Ambrosio), Salas y Gómez Island and Easter Island

\begin{tabular}{|c|c|c|c|}
\hline Lugar & Referencia & Obtención de datos & Observaciones \\
\hline \multirow[t]{6}{*}{$\begin{array}{l}\text { Islas Desventuradas } \\
\text { (San Félix y San Ambrosio) }\end{array}$} & Murphy (1936) & Febrero, 1935 & $\begin{array}{l}4 \text { hembras recolectadas por el Dr. James P. Chapin en isla San Félix. } \\
\text { Adultos no reproductivos. }\end{array}$ \\
\hline & Jehl (1973) & Junio, 1970 & $\begin{array}{l}400 \text { parejas anidando en la parte } \mathrm{SE} \text { de isla San Félix. } \\
\text { La mayoría de nidos con } 1 \text { adulto. } \\
\text { El } 20 \% \text { con } 2 \text { adultos. } \\
15 \% \text { de nidos con huevos. }\end{array}$ \\
\hline & Bahamonde (1974) & Septiembre, 1970 & $\begin{array}{l}143 \text { nidos activos. } \\
65 \text { nidos sólo con huevos. } \\
72 \text { nidos sólo con pollos. } \\
6 \text { nidos con huevos y pollos. }\end{array}$ \\
\hline & Aguirre et al. (2009) & Junio, 2001 & Presencia y defensa de nidos por adultos \\
\hline & & Diciembre, 2001 & Nidos con huevos y pollos pequeños \\
\hline & & Marzo, 2003 & Nidos con huevos y pollos en distintas etapas de desarrollo \\
\hline \multirow[t]{4}{*}{ Isla Salas y Gómez } & Harrison \& Jehl (1988) & Marzo, 1985 & $\begin{array}{l}\text { ca., } 2500 \text { parejas } \\
\text { pollos con plumón }\end{array}$ \\
\hline & Harrison \& Jehl (1988) & Marzo, 1986 & ca., 100 parejas \\
\hline & Vilina \& Gazitúa (1999) & Diciembre, 1997 & $\begin{array}{l}123 \text { adultos. } \\
92 \text { nidos activos con: } \\
66 \text { adultos con huevos } \\
25 \text { adultos con pollos } \\
1 \text { pollo solitario }\end{array}$ \\
\hline & Lazo (2011) & Febrero, 2011 & $\begin{array}{l}89 \text { adultos } \\
13 \text { juveniles } \\
8 \text { pollos }\end{array}$ \\
\hline \multirow{2}{*}{$\begin{array}{l}\text { Isla de Pascua } \\
\text { (Motu Nui) }\end{array}$} & Lazo (2011) & 2010 & $c a ., 600$ individuos* \\
\hline & Lazo (2011) & 2011 & ca., 1000 individuos* \\
\hline
\end{tabular}

*Sumatoria de conteos a lo largo del año

(1973) podrían corresponder a la subespecie Sula leucogaster etesiaca que habita desde Panamá hasta el norte de Ecuador por el Sur, incluyendo la isla Cocos (Carboneras 1992).

Este registro para Isla de Pascua, corresponde al primer registro documentado de la especie para Chile.

Considerando que esta especie se ha reportado como nidificante en islas Gambier (Thibault \& Bretagnolle 1999) y observada en islas Pitcairn, aproximadamente $2.000 \mathrm{~km}$ al oeste de Isla de Pascua (Williams 1960), es posible que el piquero café observado corresponda a Sula leucogaster plotus distribuida en el océano Índico y océano Pacífico Central (Carboneras 1992, Schreiber \& Norton 2002).

\section{Piquero blanco Sula Dactylatra (Lesson, 1831)}

El piquero blanco es la especie que presenta mayor información en la literatura científica. Ha sido reportado como especie nidificante para las islas Desventuradas (Murphy 1936, Millie 1963, Bahamonde 1966, 1974, Jehl
1973, Nelson 1978), para la isla Salas y Gómez (Schlatter 1987, Harrison \& Jehl 1988, Vilina \& Gazitúa 1999, Lazo 2011) y para Isla de Pascua (Lonnberg 1921, Jaramillo et al. 2008, Marin \& Cáceres 2010, Lazo 2011); sólo Johnson et al. (1969) lo reportan como escaso y sin nidificación para Isla de Pascua en diciembre de 1968. Además, es la única especie que cuenta con datos sobre abundancia en las islas Desventuradas (Murphy 1936, Jehl 1973, Bahamonde 1974) (Tabla 1), en isla Salas y Gómez (Harrison \& Jehl 1988, Vilina \& Gazitúa 1999, Lazo 2011) (Tabla 1) y en Isla de Pascua (Lazo 2011) (Tabla 1). Reyes $\&$ Tapia observaron un ejemplar juvenil en la costa de Cachagua (32 $35^{\prime} \mathrm{S} ; 71^{\circ} 27^{\prime} \mathrm{W}$ en agosto 2012 (Tapia com. pers.), siendo éste el registro más austral para la especie en Chile, sin embargo podría ser un ejemplar de la subespecie $S$. $d$. granti reportada para el Pacífico Este e islas Galápagos (Grace \& Anderson 2009).

La información científica disponible aún es escasa y se requiere aumentar el esfuerzo de observación de estas especies en el territorio nacional, así como generar 
proyectos de investigación de corto, mediano y largo plazo que permitan dilucidar, entre otras interrogantes, si estas especies son visitantes ocasionales, residentes, nidificantes o corresponden a registros accidentales. De igual manera, los escasos datos cuantitativos existentes, así como los años entre los registros, no permiten determinar tamaños poblacionales, ni explicar la notoria variabilidad encontrada, como se observa en el caso del piquero blanco en isla Salas y Gómez (Tabla 1).

Adicionalmente, es necesario desarrollar y estandarizar las metodologías utilizadas para realizar los estudios poblacionales y de esta manera, asegurar que los datos tomados en plataformas de oportunidad sean utilizables y contrastables con aquellos monitoreos permanentes como es el caso de las aves nidificantes en los motus de Isla de Pascua y que han sido estudiadas por personal de CONAF, siendo este monitoreo el único estudio sistemático de piqueros en las islas oceánicas chilenas.

\section{Agradecimientos}

Los autores agradecen el apoyo de PEW Environment Trust, por financiar la visita a Isla de Pascua en el marco del proyecto 'Global Ocean Legacy-Easter Island'. Asimismo, agradecemos la autorización de la Oficina Provincial de la Corporación Nacional Forestal (CONAF) para visitar el Motu Nui y al Cuerpo de Infantes de Marina de la Armada de Chile por el traslado desde Hanga Piko a Motu Nui. Agradecemos a los dos revisores anónimos que gentilmente accedieron a revisar y mejorar la calidad de este manuscrito.

\section{LITERATURA CITADA}

Aguirre JE, F Johow, H Seeger, JC Johow \& M Rubio. 2009. Nuevos registros de aves nidificantes en las islas desventuradas, Chile Insular. Boletín Chileno de Ornitología 15(1): 44-55.

Ainley D. 1980. Birds as marine organisms: a review. CalCOFI Reports 21: 48-52.

Araya B \& W Millie. 1986. Guía de campo de las aves de Chile, 389 pp. Editorial Universitaria, Santiago.

Bahamonde N. 1966. Islas Desventuradas. Museo Nacional de Historia Natural, Santiago, Serie Educativa 6: 1-15

Bahamonde N. 1974. El piquero blanco (Sula dactylatra Rothschild) de Islas Desventuradas. Noticiero Mensual, Museo Nacional de Historia Natural, Chile 18(210): 3-7.

Carboneras C. 1992. Family Sulidae (Gannets and Boobies). In: Del Hoyo J, A Elliot \& J Sargatal (eds). Handbook of the birds of the world 1: 312-325. Ediciones Lynx, Barcelona.
González-Acuña D, P Lazo, C Chávez \& K Ardiles. 2010. Piquero de patas rojas (Sula sula) en Isla de Pascua, Chile. Boletín Chileno de Ornitología 16(2): 91-94.

Grace J \& DJ Anderson. 2009. Masked Booby (Sula dactylatra), The Birds of North America Online. In: Poole A (ed). Birds of North America, Cornell Lab of Ornithology, Ithaca. 〈http://bna.birds.cornell.edu/bna/species/073>

Harrison P. 1991. Seabirds: An identification guide, 448 pp. Houghton Mifflin, Boston.

Harrison P \& JR Jehl Jr. 1988. Notes on the seabirds of Sala y Gómez. Condor 90: 259-261.

Jaramillo A. 2005. Aves de Chile, 240 pp. Lynx Edicions, Barcelona.

Jaramillo A, MTJ Johnson, CJ Rothfels \& RA Johnson. 2008. La avifauna nativa y exótica de Isla de Pascua: antes y ahora. Boletín Chileno de Ornitología 14(1): 8-21.

Jehl JR Jr. 1973. The distribution of marine birds in Chilean waters in winter. The Auk 90: 114-135.

Johnson AW, WR Millie \& G Moffett. 1969. Notes on the birds of Easter Island. Ibis 112(4): 532-538.

King WB. 1967. Seabirds of the Tropical Pacific Ocean. Preliminary Smithsonian identification mannual, 126 pp. Smithsonian Institution, Washington.

Lazo P. 2011. Informe final: censo y monitoreo de avifauna en el Parque Nacional Rapa Nui 2011. Informe Técnico, Corporación Nacional Forestal (CONAF), Santiago, pp. $1-41$.

Lonnberg E. 1921. Notes on the birds of Easter Island. In: Skottsberg C (ed). The natural history of Juan Fernández and Easter Island, pp 19-24. Alqvist \& Wiksells Boktryckeri A-B, Uppsala.

Marin M \& P Cáceres. 2010. Sobre las aves de Isla de Pascua. Boletín, Museo Nacional de Historia Natural, Chile 59: 7595.

Martínez D \& G González. 2004. Las aves de Chile. Nueva guía de campo, 620 pp. Ediciones del Naturalista, Santiago.

Millie WR. 1963. Brief notes on the birds of San Ambrosio and San Félix Islands, Chile. Ibis 105(4): 563-566.

Millie W, B Araya \& O Magnére. 1973. Un piquero nuevo para los mares chilenos: Sula leucogaster (Boddaert, 1783). Noticiario Mensual, Museo Nacional de Historia Natural, Chile 200: 9-10.

Murphy R. 1936. Oceanic birds of South America 2. The American Museum of Natural History New York, 680 pp. The MacMillan Company, New York.

Nelson JB. 1978. The Sulidae -Gannets and Boobies, 1012 pp. Oxford University Press, Oxford.

Nelson JB. 2005. Pelicans, Cormorants, and their relatives: The Pelecaniformes, 677 pp. Oxford University Press, Oxford.

Schlatter RP. 1984. The status and conservation of seabirds in Chile. ICBP Technical Publication 2: 261-269. 
Schlatter RP. 1987. Conocimiento y situación de la ornitofauna en las islas oceánicas chilenas. In: Castilla JC (ed). Islas oceánicas chilenas: conocimiento científico y necesidades de investigaciones, pp. 271-285. Ediciones Universidad Católica de Chile, Santiago.

Schreiber EA \& RL Norton. 2002. Brown Booby (Sula leucogaster). The Birds of North America Online. In: Poole A (ed). Birds of North America. Cornell Lab of Ornithology. Ithaca. <http://bna.birds.cornell.edu/bna/species/649 >

Schreiber EA, RW Schreiber \& GA Schenk. 1996. Redfooted Booby (Sula sula). The Birds of North America Online. In: Poole A (ed.). Birds of North America. Cornell Lab of Ornithology, Ithaca. <http://bna.birds.cornell.edu/ bna/species/241>

Shallenberger RJ, GC Whittow \& RM Smith. 1974. Bodytemperature of nesting Red-footed Booby (Sula sula). Condor 76: 476-478.
Thibault J-C \& V Bretagnolle. 1999. Breeding seabirds of Gambier Island, Easter Polynesia: Numbers and Changes during the $20^{\text {th }}$ Century. EMU 99: 100-107.

Valverde M. 2007. Registro del Piquero Pardo Sula leucogaster en una isla del Perú. Biologist, Lima 5(2): 65-67.

Vilina Y \& F Gazitúa. 1999. The birds of Salas y Gómez Island, Chile. Waterbirds 22(3): 459-462.

Weimerskirch H, M Le Corre, Y Ropertcoudert, A Kato \& F Marsac. 2005. The three-dimensional flight of red-footed boobies: adaptations to foraging in a tropical environment? Proceedings of the Royal Society B 272: 53-61.

Williams GR. 1960. The birds of the Pitcairn Islands, Central South Pacific Ocean. Ibis 102: 58-70.

Recibido el 16 de enero de 2013 y aceptado el 3 de octubre de 2013

Editor: Claudia Bustos D. 